\title{
Comparison between radial artery tonometry pulse analyzer and pulsed-Doppler echocardiography derived hemodynamic parameters in cardiac surgery patients- a pilot study
}

\author{
Rashad Zayat ${ }^{\text {Corresp.. }}{ }^{1}$, Andreas Goetzenich ${ }^{1}$, Ju-Yeon Lee ${ }^{2}$, HeeJung Kang ${ }^{3}$, So-Hyun Jansen-Park ${ }^{2}$, Thomas

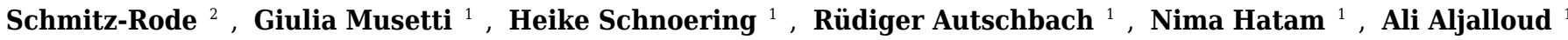 \\ ${ }^{1}$ Department of Thoracic and Cardiovascular Surgery, RWTH University Hospital Aachen, Aachen, Germany \\ 2 Institute of Applied Medical Engineering, Helmholtz Institute, RWTH Aachen University, Aachen, Germany \\ 3 DAEYOMEDI Co. Ltd., Ansan-Si, Gyeonggi-do, South Korea \\ Corresponding Author: Rashad Zayat \\ Email address: rzayat@ukaachen.de
}

Background: Bedside non-invasive techniques, such as radial artery tonometry, to estimate hemodynamic parameters have gained increased relevance as an attractive alternative and efficient method to measure hemodynamics in outpatient departments. For our pilot study, we sought to compare cardiac output (CO), and stroke volume (SV) estimated from a radial artery tonometry blood pressure pulse analyzer (BPPA) (DMP-Life, DAEYOMEDI Co., South Korea) to pulsed-wave Doppler (PWD) echocardiography derived parameters. Methods: From January 2015 to December 2016, all patients scheduled for coronary artery bypass (CABG) surgery at our department were screened. Exclusion criteria were, inter alia, moderate to severe aortic- or Mitral valve disease and peripheral arterial disease (PAD) > stage II. 107 patients were included (mean age 66.1 \pm 9.9, 15 females, mean BMI $27.2 \pm 4.1 \mathrm{~kg} / \mathrm{m}^{2}$ ). All patients had pre-operative transthoracic echocardiography (TTE). We measured the hemodynamic parameters with the BPPA from the radial artery, randomly before or after TTE. For the comparison between the measurement methods we used the Bland-Altman test and Pearson correlation. Results: Mean TTE-CO was $5.1 \pm 0.96 \mathrm{~L} / \mathrm{min}$., and the mean BPPA-CO was $5.2 \pm 0.85 \mathrm{~L} / \mathrm{min}$. The Bland-Altman analysis for CO revealed a bias of $-0.13 \mathrm{~L} / \mathrm{min}$ and SD of $0.90 \mathrm{~L} / \mathrm{min}$ with upper and lower limits of agreement of -1.91 and $+1.64 \mathrm{~L} / \mathrm{min}$. The correlation of $\mathrm{CO}$ measurements between DMP-life and TTE was poor $(r=0.501, p<0.0001)$. The mean TTE-SV was $71.3 \pm 16.2 \mathrm{~mL}$ and the mean BPPA-SV was $73.8 \pm 19.2 \mathrm{~mL}$. SV measurements correlated very well between the two methods $(r=0.900, p<0.0001)$. The Bland-Altman analysis for SV revealed a bias of $-2.54 \mathrm{~mL}$ and SD of $\pm 8.42 \mathrm{~mL}$ and upper and lower limits of agreement of -19.05 and $+13.96 \mathrm{~mL}$, respectively. Conclusion: Our study shows for the first time that the DMP-life tonometry device measures SV and CO with reasonable accuracy and precision of agreement compared with TTE in preoperative cardiothoracic 
surgery patients. Tonometry BPPA are relatively quick and simple measuring devices, which facilitate the collection of cardiac and hemodynamic information. Further studies with a larger number of patients and with repeated measurements are in progress to test the reliability and repeatability of DMP-Life system. 
1 Comparison between radial artery tonometry pulse analyzer and pulsed-Doppler

2 echocardiography derived hemodynamic parameters in cardiac surgery patients-

3 a pilot study

4 Rashad Zayat ${ }^{1^{*}}$, Andreas Goetzenich ${ }^{1}$, Ju-Yeon Lee ${ }^{2}$, HeeJung Kang ${ }^{3}$, So-Hyun

5 Jansen-Park ${ }^{2}$, Thomas Schmitz-Rode ${ }^{2}$, Giulia Musettii ${ }^{1}$, Heike Schnoering ${ }^{1}$, Rüdiger

6 Autschbach $^{1}$, Nima Hatam ${ }^{1+}$, Ali Aljalloud ${ }^{1+}$

7 1: Department of Thoracic and Cardiovascular Surgery, RWTH University Hospital,

8 Aachen, Germany

9 2: Department of Cardiovascular Engineering, Institute of Applied Medical Engineering,

10 Helmholtz Institute, RWTH Aachen University, Aachen, Germany.

11 3: DAEYOMEDI Co. Ltd., Ansan-Si, Gyeonggi-do, South Korea.

12 + both authors contributed equally last

13

$14{ }^{*}$ Correspondence:

15 Rashad Zayat, MD

16 Department of Thoracic and Cardiovascular Surgery

17 RWTH University Hospital

18 Pauwelsstr. 30

1952078 Aachen, Germany

20 E-Mail: rzayat@ukaachen.de (RZ) 


\section{Abstract:}

23

Background: Bedside non-invasive techniques, such as radial artery tonometry, to estimate hemodynamic parameters have gained increased relevance as an attractive alternative and efficient method to measure hemodynamics in outpatient departments. For our pilot study, we sought to compare cardiac output (CO), and stroke volume (SV) estimated from a radial artery tonometry blood pressure pulse analyzer (BPPA) (DMPLife, DAEYOMEDI Co., South Korea) to pulsed-wave Doppler (PWD) echocardiography derived parameters.

Methods: From January 2015 to December 2016, all patients scheduled for coronary artery bypass (CABG) surgery at our department were screened. Exclusion criteria were, inter alia, moderate to severe aortic- or Mitral valve disease and peripheral arterial disease $(P A D)>$ stage II. 107 patients were included (mean age 66.1 $\pm 9.9,15$ females, mean BMI $27.2 \pm 4.1 \mathrm{~kg} / \mathrm{m}^{2}$ ). All patients had pre-operative transthoracic echocardiography (TTE). We measured the hemodynamic parameters with the BPPA from the radial artery, randomly before or after TTE. For the comparison between the measurement methods we used the Bland-Altman test and Pearson correlation.

Results: Mean TTE-CO was $5.1 \pm 0.96 \mathrm{~L} / \mathrm{min}$., and the mean BPPA-CO was $5.2 \pm 0.85$ $\mathrm{L} / \mathrm{min}$. The Bland-Altman analysis for $\mathrm{CO}$ revealed a bias of $-0.13 \mathrm{~L} / \mathrm{min}$ and $\mathrm{SD}$ of 0.90

$\mathrm{L} / \mathrm{min}$ with upper and lower limits of agreement of -1.91 and $+1.64 \mathrm{~L} / \mathrm{min}$. The

42 correlation of CO measurements between DMP-life and TTE was poor $(r=0.501, p<$ 3 0.0001). The mean TTE-SV was $71.3 \pm 16.2 \mathrm{~mL}$ and the mean BPPA-SV was $73.8 \pm$ $4419.2 \mathrm{~mL}$. SV measurements correlated very well between the two methods $(r=0.900, p$ $45<0.0001)$. The Bland-Altman analysis for SV revealed a bias of $-2.54 \mathrm{~mL}$ and SD of \pm 
$468.42 \mathrm{~mL}$ and upper and lower limits of agreement of -19.05 and $+13.96 \mathrm{~mL}$,

47 respectively.

48 Conclusion:

49 Our study shows for the first time that the DMP-life tonometry device measures SV and

50 CO with reasonable accuracy and precision of agreement compared with TTE in

51 preoperative cardiothoracic surgery patients. Tonometry BPPA are relatively quick and

52 simple measuring devices, which facilitate the collection of cardiac and hemodynamic

53 information. Further studies with a larger number of patients and with repeated

54 measurements are in progress to test the reliability and repeatability of DMP-Life

55 system.

56

57 
58 Introduction:

59 Hemodynamic monitoring is crucial, not only during anesthesia and in intensive care

60 units (ICU) but also in the normal ward and in the follow-up during outpatient visits,

61 especially in cardiac surgery patients. The main goal of hemodynamic monitoring is to

62 ensure a sufficient end organ perfusion and oxygen delivery by optimizing stroke

63 volume (SV) and cardiac output (CO). The gold standard in monitoring $\mathrm{CO}$ and SV is

64 still the pulmonary artery catheter (PAC), its invasive nature and life-threatening

65 complications largely restrict its use in the operating room and in the ICU (Marik 2013;

66 Wheeler et al. 2006). During the last decade, several non- or minimally invasive

67 techniques have been presented, such as pulse wave transit time, non-invasive pulse

68 contour analysis, arterial tonometry, oscillometry, esophageal Doppler devices, the

69 partial carbon dioxide rebreathing technique, and transthoracic electrical bio-impedance

70 measurements. However, PAC is still the most accepted method as there is still no

71 clearly established gold standard for CO measurement in human studies (Peyton \&

72 Chong 2010; Sato et al. 1993; Thiele et al. 2015). Concurrently, both transesophageal

73 (TEE) and transthoracic (TTE) echocardiography, have become a frequently utilized

74 monitor in the cardiac operating rooms and ICU. TEE/TTE derived SV and CO have

75 been validated against PAC-based values (Harris et al. 1999; Perrino et al. 1998).

76 TTE/TEE have many advantages, but there are some limitations, such as the need for

77 an accurate ultrasound window, which is not always possible post sternotomy, semi-

78 continuous real-time monitoring, and operator dependence. Lately, radial artery

79 tonometry with the use of a piezoresistive array sensor has been presented as a non-

80 invasive method to monitor the blood pressure and analyze the radial artery waveform 
81 (Jun et al. 2016; Shin et al. 2010). In this pilot study, we aimed to compare the CO and

82 SV estimated from a new radial tonometry blood pressure pulse analyzer (BPPA) using

83 the piezoresistive array sensor array technique (DMP-Life, DAEYOMEDI Co., Ltd.,

84 Gyeonggi-do, South Korea) with the TTE derived CO and SV in cardiac surgery patients

85 prior to surgery.

86

87 Materials and Methods:

88 Patients and data collection:

89 Our study was conducted in the cardio-surgical department of a German university

90 hospital (RWTH University Hospital, Aachen, Germany). The ethics committee

91 (Ethikkommission der RWTH Aachen) approved the study (EK 151/90). Written

92 informed consent was obtained from all patients prior to study enrolment.

93 Between January 2015 and December 2016, all patients who were planned for

94 nonemergency coronary artery bypass graft (CABG) surgery, were screened. Exclusion

95 criteria were: 1) peripheral artery disease (PAD) more than Fontaine grade II, 2)

96 moderate to severe aortic stenosis or regurgitation, 3) moderate to severe mitral

97 stenosis or regurgitation, 4) atrial fibrillation, 5) left ventricular ejection fraction $<40 \%, 6$ )

98 no accurate ultrasound window for TTE. 572 patients were screened and 107 patients

99 were included in the study (Figure 1). Patients' data were prospectively entered to our

100 institution's electronic database.

101

102 Echocardiographic and Tonometry measurements: 
103 After enrolment in the study, all patients had TTE examinations 2 to 3 days prior to the

104 scheduled CABG. TTE studies were performed using a commercially available

105 machine (Vivid E9® Vingmed-General Electrics, Horten, Norway), and data analysis

106 was performed offline using the Echopac system. TTE were performed according to the

107 American society of echocardiography and European association for echocardiography

108 (ASE/EAE) guidelines (Lang et al. 2015). All TTE studies and measurements with the

109 DMP-Life were performed in the same room, under standardized conditions and by the

110 same operators for the entire cohort. TTE examinations were performed in a left lateral

111 position. Measurements with DMP-life were obtained in a lying supine position from the

112 left or right radial artery, randomly. The average of DMP-life measurements from at

113 least 5 heart beats were calculated. The two measurements (TTE and DMP-life) were

114 performed in random order.

115 TTE derived SV was then calculated using the pulsed Doppler (PW) through the left

116 ventricular outflow tract (LVOT) as a product of the velocity time integral through the

117 LVOT and the cross-sectional area of the LVOT as described by Lewis et al. (Lewis et

118 al. 1984). The stroke volume (SV) was calculated by measuring: 1) the diameter (d) of

119 the LVOT from the parasternal window was used to calculate the cross-sectional area

$\left.120(\mathrm{CSA})\left[\mathrm{CSA}_{L V O T}=(d / 2)^{2} \times \pi\right] ; 2\right)$ the velocity time integral $(\mathrm{VTI})$ measured the same

121 site $\left(\mathrm{LVOT}_{\mathrm{VTI}}\right)$ at the apical three-chamber view with pulsed-wave Doppler. SV averaged

122 over five consecutive beats was used to compute the CO (SV $\times$ heart rate).

123

124 DMP-Life system description: 
125 The pulse was measured by radial artery tonometry device DMP-Life (DAEYOMEDI

126 Co., South Korea). With the array pressure sensor equipment, tonometry devices have

127 improved to confirm blood vessels' position accurately and also improved reproducibility

128 and reliability. Through orthogonal applanation to radial artery, tonometry devices can

129 get signals, which include blood pressure, skin condition, blood vessel stiffness and

130 hemodynamic condition (Jun et al. 2016; Singh et al. 2017; Wagner et al. 2016).

131 The sensor module of DMP-Life is equipped with multi-channel array with 5

132 piezoresistive semiconductor transducers (Figure 2A) and precise moving actuator to

133 apply pressure automatically by a given algorithm. During pressure application on radial

134 artery, the actuator moves very slowly to avoid any fluctuation on the signal baseline, to

135 have a maximum control resolution of $12 \mu \mathrm{m}$, and to detect accurate skin property and

136 blood pressure (Figure 2C).

137 The range of the applied pressure in DMP-Life system is set at $0 \sim 400 \mathrm{g.f} / \mathrm{cm}^{2}$, but for

138 each patient, individual maximum applied pressure can vary according to patient's pulse

139 pressure response. Waveforms at each applying pressure can be used to analyze the

140 elasticity and stiffness of the vascular system. The concept of pulse pressure (systolic

141 blood pressure minus the diastolic pressure) is applied to the tonometric sensor.

142 In DMP-life, the difference between the peak voltage and the lowest voltage measured

143 in one cycle is defined as a pulse force. Figure 3 shows some cases of measured pulse

144 forces for each applying pressure. For example, in Figure 3A the maximum pulse height

145 in this patient is reached when a force of $150 \mathrm{~g} . f / \mathrm{cm}^{2}$ is applied, and in Figure 3D the

146 patient's maximum pulse height is first reached when a pressure of $260 \mathrm{~g} . \mathrm{f} / \mathrm{cm}^{2}$ was

147 applied. The response of the pulse to the applied pressure result in a typical shape for 
148 each patient. Sharp end or arrow shape or triangle shape may appear in healthy elastic

149 vessel (Figure 3A, B and D), but smooth and flat curved end similar to half-arc shape

150 may result due to stiff or tensed vessel (Figure 3C). The correlation of different shapes

151 due to individual vessels response with the severity of cardiovascular disease is still

152 under investigation.

153 The multi-channel array with 5 piezoresistive scan the radial pulse location to identify

154 the site of maximum pulsation. The pulse pressure amplitude varies least at distances

155 furthest from the vessel to greatest at the optimal position over the pulse. Thick skin,

156 deep arteries and high mean artery blood pressure can be a cause of stronger pressure

157 and long measurement time, because the actuator moves in constant velocity during

158 detection of the pulse signal. The measured pulse signal is analyzed by a patented

159 algorithm, which is a modified algorithm based on the systolic area with Kouchoukos

160 correction algorithm (Kouchoukos et al. 1970) and the algorithm hold a patent from the

161 Korean Intellectual Property Office (Patent number: 100785901000, 1006948960000).

162 The measured data with the DMP-Life system are: systolic blood pressure (SBP),

163 diastolic blood pressure (DBP), pulse pressure (PP), pulse rate (PR), radial-augmented

164 index (R-Al), stroke Volume (SV), stroke volume index (SVI), cardiac output (CO),

165 cardiac index $(\mathrm{Cl})$, systemic circulation resistance index (SCRI) and pulse conditions.

166 For this pilot study, we used only SV and CO.

167 DMP-Life uses a modified systolic area with Kouchoukos correction algorithm

168 (Kouchoukos et al. 1970) to calculate SV with measured waveform parameter.

$169 S V=a+b \times(T 4+c)+d \times P R+e \times P P+f \times B S A+g \times A g e ;$ and $C O=S V \times P R$,

170 where, T4: LV (sec); PR: Pulse rate (beat/min); PP: Pulse Pressure (mmHg); BSA: 
171 Body surface area $\left(\mathrm{m}^{2}\right) ; \mathrm{a}, b, c, d, e, f$ and $g$ : coefficient constants. DMP-life has

172 tonometric blood pressure measure function and does not need calibration with other

173 blood pressure monitor.

174 Measurements with the DMP-life system:

175 Before performing the measurement with DMP-Life, patients should take at least 5

176 minutes of rest time. Patients were in lying supine position. After entering the patient's

177 biometric data (height, weight, age, and gender), the physician then first felt the radial

178 artery pulse and chose the best position. A bracelet with the pressure sensor cartridge

179 (DMP-life) was placed on the patient's wrist over the radial artery (Figure 2D). The

180 sensor of DMP-life includes multi-channel array with 5 piezoresistive semiconductor

181 transducers (Figure 2A). When the physician turns the device on, the device software

182 automatically judges and gives notice to the operator whether the sensor module is

183 positioned well or not. When the sensor position is confirmed, the actuator moves in

184 constant velocity and applies pressure on the radial artery automatically by a given

185 algorithm to partially flatten the radial artery (Figure $2 \mathrm{C}$ ). The radial artery pressure is

186 then transmitted from the vessel to the sensor and is recorded digitally. When the

187 optimal signal is available, the DMP-life monitor provides a continuous arterial waveform

188 (Figure 4A). The quality of the captured pulse waves can be visually assessed by the

189 physician on the monitor and also the device performs default quality check: 1.) The

190 system automatically confirms vessel position when the array sensor starts capturing

191 the pulse waves. If the sensor position is out of vessel's area then the system gives a

192 note and ask the operator to repeat the measurements, 2) The system automatically

193 detect applied pressure and corresponding changes in pulse waves. If inappropriate 
194 pulse wave signals are captured with abnormal vessels respond, the system quit

195 measuring and ask for repeating the measurements.

196 The DMP-life system's monitor displays inter alia CO and SV values derived from pulse

197 contour analysis using a proprietary auto-calibrating algorithm, as mentioned above

198 (Figure 4B).

199

200 Statistical analysis

201 Continuous variables are presented as mean \pm standard deviation (SD) or as median

202 (25 th and $75^{\text {th }}$ percentile), where appropriate. Categorical variables are described in

203 absolute numbers and percentages. We computed the mean of the differences (= bias)

204 between BPPA- and TTE-CO/SV, the SD, and the $95 \%$ limits of agreement (= bias \pm

$2051.96 \times \mathrm{SD})$ to describe the agreement between BPPA (DMP-life) and TTE

206 measurements, Bland-Altman plots for repeated measures were also calculated (Bland

207 \& Altman 2007). The Pearson product moment correlation test was used to evaluate the 208 correlation of SV and CO between the two methods (DMP-life and TTE). All statistical

209 analyses were performed using IBM SPSS Statistics 23 (SPSS, Chicago, IL).

210

\section{Results:}

212 Patients characteristics:

213107 patients were included in the study. The mean age was $66.1 \pm 9.9$ years and $14.1 \%$

214 were female. Clinical characteristics of the patients are demonstrated in Table 1. Drug

215 use was: beta blockers (96\%), angiotensin-converting enzyme inhibitors 
216 and/or angiotensin 2 receptor blockers (85\%), diuretics (78\%), spironolactone (10\%),

217 statins (68\%) and aspirin (98\%), respectively.

218 SV and CO measurements:

At the time of performing the measurements with TTE and DMP-life, the mean systolic blood pressure was $136.9 \pm 11.6 \mathrm{mmHg}$, mean diastolic blood pressure was $82.2 \pm 6.2$

$222 \mathrm{mmHg}$ and the heart rate was $71.8 \pm 15.1 / \mathrm{min}$.

223 A total of 107 matched SV and CO data points were available for the final statistical 224 analysis.

225 The mean TTE-SV was $71.3 \pm 16.2 \mathrm{~mL}$ and the mean BPPA-SV was $73.8 \pm 19.2 \mathrm{~mL}$.

226 The Bland-Altman analysis for SV revealed a bias of $-2.54 \mathrm{~mL}$ and $\mathrm{SD}$ of $\pm 8.42 \mathrm{~mL}$ and

227 upper and lower limits of agreement of -19.05 and $+13.96 \mathrm{~mL}$, respectively (Figure $5 \mathrm{~A}$ ).

228 SV measurements correlated very well between the two methods, DMP-life and TTE, $(r$

$229=0.900, p<0.0001$ ) and the $r$ squared $\left(r^{2}\right)$ for the goodness of fit was 0.811 (Figure

230 5B). The mean TTE-CO was $5.1 \pm 0.96 \mathrm{~L} / \mathrm{min}$. and the mean BPPA-CO was $5.2 \pm 0.85$

$231 \mathrm{~L} / \mathrm{min}$. The Bland-Altman analysis for $\mathrm{CO}$ revealed a bias of $-0.13 \mathrm{~L} / \mathrm{min}$ and $\mathrm{SD}$ of 0.90

$232 \mathrm{~L} / \mathrm{min}$ with upper and lower limits of agreement of -1.91 and $+1.64 \mathrm{~L} / \mathrm{min}$ (Figure $5 \mathrm{C}$ ).

233 Pearson correlation demonstrated a poor correlation of the measured $\mathrm{CO}$ in the two

234 methods $\left(r=0.501, p<0.0001, r^{2}=0.251\right)$ (Figure 5D).

235

236 Discussion:

237 Our study shows for the first time that the DMP-life technology measures SV and CO

238 with reasonable accuracy and precision of agreement compared with TTE in

239 preoperative cardiothoracic surgery patients. Our findings are promising as there is a 
240 clear trend toward non-invasive hemodynamic monitoring (Saugel \& Reuter 2014;

241 Vincent et al. 2011). The bias for SV and CO between DMP-life and TTE were -2.54 mL

242 and $-0.13 \mathrm{~L} / \mathrm{min}$, respectively, which is acceptable in the clinical context but definitive

243 recommendations for the definition of clinical acceptable agreement between two CO

244 measuring systems are still under debate (Saugel \& Reuter 2014; Wagner et al. 2015).

245 The Pearson correlation coefficient was excellent for the SV.

246 On the contrary, CO correlation was poor. As CO is calculated from SV and heart rate,

247 this discordance can only be explained by a variation in patient's heart rate. As TTE and

248 DMP-life were not performed simultaneously, variation in the heart rate was present in

249 all patients, explaining this result. PAC is still used as the reference method to monitor

250 hemodynamics and to validate alternative monitoring systems (Rajaram et al. 2013), but

251 its invasive nature and life-threatening complications largely restrict its use in the

252 operating room and in the ICU (Marik 2013; Wheeler et al. 2006). Echocardiography

253 has been well accepted as a diagnostic tool for circulatory failure and as an alternative

254 for PAC to evaluate hemodynamic parameters (De Backer 2014; Porter et al.

255 2015). Measuring hemodynamics with TTE has some limitations: the need of trained

256 sonographer, poor acoustic window, inaccurate diameter calculations, and difficulty

257 maintaining the angle of insonation (2014; Narasimhan et al. 2014). Although the PAC

258 still the gold standard, echocardiography has been used to test newer hemodynamic

259 monitoring systems (Gola et al. 1996; Romagnoli et al. 2013; Scolletta et al. 2016) and it

260 has been recommended by international consensus as a reliable method for $\mathrm{CO}$

261 estimation (Cecconi et al. 2014). During the last decade, several non- or minimal

262 invasive techniques have been presented, such as pulse wave transit time, non- 
263 invasive pulse contour analysis, arterial tonometry, oscillometry, oesophageal Doppler

264 devices, the partial carbon dioxide rebreathing technique, and transthoracic electrical

265 bio-impedance (Peyton \& Chong 2010; Sato et al. 1993; Thiele et al. 2015). Our results

266 and comparison are similar to De Castro et al. (De Castro et al. 2006), who used aortic

267 Doppler echocardiography to compare the estimated SV from an axillary artery pulse-

268 contour and found that there is a good correlation between pulse-contour analysis and

269 aortic Doppler from transesophageal echocardiography $(r=0.842, p<0.0001)$.

270 Romangoli et al. (Romagnoli et al. 2013) also analyzed TTE-CO in comparison with two

271 different pulse contour devices. In a multicenter study by Scolleta et al. (Scolletta et al.

272 2016) Doppler TTE was used as a comparison method to pulse contour device, and

273 they could demonstrate a good correlation between TTE-CO and the CO estimated

274 from a pulse contour device $(r=0.85 ; p<0.0001)$. Our findings are in line with Wagner

275 et al. (Wagner et al. 2015) who demonstrated that the applanation tonometry technology

276 provides $\mathrm{CO}$ values with reasonable accuracy and precision of agreement compared

277 with intermittent pulmonary artery thermodilution measurements. The mean of

278 differences in the study by Wagner et al. (Wagner et al. 2015) was $-0.2 \mathrm{~L} / \mathrm{min}$ with $95 \%$

279 limits of agreement of -1.8 to $+1.4 \mathrm{~L} / \mathrm{min}$. The percentage error was $34 \%$.

280 On the other hand Compton et al. (Compton et al. 2008) compared radial artery

281 tonometry to invasive estimated $\mathrm{CO}$ with $\mathrm{PICCO} \circledast$ transpulmonary thermodilution or

282 with PAC in 49 critically ill medical ICU patients and they concluded that radial artery

283 applanation tonometry is not suitable to determine $\mathrm{CO}$ in critically ill hemodynamically

284 unstable patients. 
285 Arterial tonometry is a non-invasive method for blood pressure measurement and it

286 provides complete pulse pressure waveform, which in turn has implications in several

287 disease diagnoses (Laurent et al. 2006; Nelson et al. 2010).

288 A typical arterial tonometer consists of a plunger and pressure sensor placed at the

289 centre of a superficial artery. The plunger is used for arterial flattening via the

290 application of hold down pressure. A pressure sensor is used to measure contact

291 stresses at the surface above the radial artery at optimal level of flattening (Singh et al.

292 2017). Different types of sensing elements in tonometer systems for effective pulse

293 measurements and analyses, such as piezoelectric, piezoresistive, capacitive, and hall

294 sensors, have been recently presented (Hu et al. 2012; Liu \& Tyan 2010). DMP-Life is

295 equipped with multi-channel array with 5 piezoresistive semiconductor transducers and

296 with a precise moving actuator to apply pressure automatically by specified algorithm.

297 While applying the pressure on a radial artery, the actuator moves very slowly to avoid

298 any fluctuation on signal baseline and to provide maximum control resolution with $12 \mu \mathrm{m}$

299 to detect accurate skin properties and blood pressure (Fig 2C). The piezoresistive

300 sensor is the most precise method for measuring radial artery pulses because it can

301 collect static and dynamic information on pulse waves with high sensitivity (Jun et al.

302 2016). The interaction between the shape of the plunger and the geometry of the wrist

303 and the arrangement of the sensor array play a key role in precisely collecting and

304 analyzing pulse wave signals (Jun et al. 2016; Singh et al. 2017). The concave shape of

305 the DMP-life's plunger and the arrangement of 5 piezoresistive sensors provide

306 accurate interaction with the wrist geometry and allows estimating the optimal blood

307 vessel direction as, when a sensor is at the center of the radial artery, it has a high- 
308 amplitude signal, and the signal becomes smaller as a sensor is far from the center.

309 The DMP-Life filters automatically the signals from all five channels and chooses the

310 optimum signal. Our stroke volume estimation is based on blood pressure, body surface

311 area, and systolic time.

312 The DMP-Life pressure pulse analyzer is a relatively quick and simple measuring

313 method, which facilitates the collection of cardiac and hemodynamic information.

314 Since the tonometry technique of estimating the $\mathrm{CO}$ and SV is relatively accurate, it

315 seems to be a good tool for patients with cardiac disease who need continuous self-

316 monitoring.

318 Limitations of the Study:

319 Our study is limited by the usual shortcomings of a small cohort single-center study and

320 a probably heterogeneous patient group. In our proof of concept study, we did not use

321 PAC as invasive method to compare CO and SV estimated from DMP-Life, which may

322 limit the interpretation of the accuracy of DMP-life measurements, on the other hand,

323 TTE measurements have been validated against PAC based values. A major issue

324 related with the accuracy of CO/SV measurements is the reproducibility of the

325 measurement, which was not assessed in our study and no repeated measurements in

326 pre, peri and postoperative period were performed to determine whether DMP-Life

327 detect changes in $\mathrm{CO}$ precisely. Another important issue was that measurements with

328 DMP-life and TTE were not performed simultaneously, which did result in a poor

329 correlation of $\mathrm{CO}$, most likely due to different inter-individual heart rate at the time of

330 performing the measurements. 
331 One of the limitation of tonometry method is that the signal is very position sensitive, the

332 transducer needs to be positioned directly over the center of the artery. This has been

333 dealt with by using an array of 5 piezoresistive transducers placed across the artery. 334

335 Conclusion:

336 Our study demonstrates for the first time that the DMP-life tonometry device measures

337 SV and CO with reasonable accuracy and precision of agreement compared with TTE

338 in preoperative cardiothoracic surgery patients. Tonometry BPPAs are relatively quick

339 and simple measuring devices, which facilitates the collection of hemodynamic

340 information. Further studies with a larger number of patients with repeated

341 measurements are in progress to test the reliability and repeatability of DMP-Life

342 system measurements. 


\section{Acknowledgments}

345 Not applicable.

346 
347 References:

348 2014. International consensus statement on training standards for advanced critical care echocardiography. Intensive Care Med 40:654-666. 10.1007/s00134-014$3228-5$

351 Bland JM, and Altman DG. 2007. Agreement between methods of measurement with multiple observations per individual. J Biopharm Stat 17:571-582. $10.1080 / 10543400701329422$

354 Cecconi M, De Backer D, Antonelli M, Beale R, Bakker J, Hofer C, Jaeschke R, Mebazaa A, Pinsky MR, Teboul JL, Vincent JL, and Rhodes A. 2014. Consensus on circulatory shock and hemodynamic monitoring. Task force of the European Society of Intensive Care Medicine. Intensive Care Med 40:1795-1815. $10.1007 / \mathrm{s} 00134-014-3525-\mathrm{z}$

Compton F, Wittrock M, Schaefer JH, Zidek W, Tepel M, and Scholze A. 2008. Noninvasive cardiac output determination using applanation tonometry-derived radial artery pulse contour analysis in critically ill patients. Anesth Analg 106:171174, table of contents. 10.1213/01.ane.0000297440.52059.2c

363 De Backer D. 2014. Ultrasonic evaluation of the heart. Curr Opin Crit Care 20:309-314. 10.1097/mcc.0000000000000094

365 De Castro V, Goarin JP, Lhotel L, Mabrouk N, Perel A, and Coriat P. 2006. Comparison of stroke volume (SV) and stroke volume respiratory variation (SVV) measured by the axillary artery pulse-contour method and by aortic Doppler echocardiography in patients undergoing aortic surgery. Br J Anaesth 97:605610. $10.1093 /$ bja/ael236 
370 Gola A, Pozzoli M, Capomolla S, Traversi E, Sanarico M, Cobelli F, and Tavazzi L.

371 1996. Comparison of Doppler echocardiography with thermodilution for

372 assessing cardiac output in advanced congestive heart failure. Am J Cardiol

$373 \quad 78: 708-712$.

374 Harris SN, Luther MA, and Perrino AC, Jr. 1999. Multiplane transesophageal

375 echocardiographic acquisition of ascending aortic flow velocities: A comparison

376 with established techniques. J Am Soc Echocardiogr 12:754-760.

377 Hu CS, Chung YF, Yeh CC, and Luo CH. 2012. Temporal and spatial properties of

378 arterial pulsation measurement using pressure sensor array. Evid Based

$379 \quad$ Complement Alternat Med 2012:745127. 10.1155/2012/745127

380 Jun MH, Kim YM, Bae JH, Jung CJ, Cho JH, and Jeon YJ. 2016. Development of a

381 Tonometric Sensor with a Decoupled Circular Array for Precisely Measuring

382 Radial Artery Pulse. Sensors (Basel) 16. 10.3390/s16060768

383 Kouchoukos NT, Sheppard LC, and McDonald DA. 1970. Estimation of stroke volume in

384 the dog by a pulse contour method. Circ Res 26:611-623.

385 Lang RM, Badano LP, Mor-Avi V, Afilalo J, Armstrong A, Ernande L, Flachskampf FA,

386 Foster E, Goldstein SA, and Kuznetsova T. 2015. Recommendations for cardiac

387 chamber quantification by echocardiography in adults: an update from the

$388 \quad$ American Society of Echocardiography and the European Association of

389 Cardiovascular Imaging. Journal of the American Society of Echocardiography

$390 \quad$ 28:1-39. e14.

391 Laurent S, Cockcroft J, Van Bortel L, Boutouyrie P, Giannattasio C, Hayoz D, Pannier

392 B, Vlachopoulos C, Wilkinson I, and Struijker-Boudier H. 2006. Expert consensus 
document on arterial stiffness: methodological issues and clinical applications. European Heart Journal 27:2588-2605. 10.1093/eurheartj/ehl254

Lewis JF, Kuo LC, Nelson JG, Limacher MC, and Quinones MA. 1984. Pulsed Doppler echocardiographic determination of stroke volume and cardiac output: clinical validation of two new methods using the apical window. Circulation 70:425-431.

Liu S-H, and Tyan C-C. 2010. Quantitative analysis of sensor for pressure waveform measurement. BioMedical Engineering OnLine 9:6. 10.1186/1475-925x-9-6

Marik PE. 2013. Obituary: pulmonary artery catheter 1970 to 2013. Ann Intensive Care 3:38. 10.1186/2110-5820-3-38

Narasimhan M, Koenig SJ, and Mayo PH. 2014. Advanced echocardiography for the critical care physician: part 1. Chest 145:129-134. 10.1378/chest.12-2441

Nelson MR, Stepanek J, Cevette M, Covalciuc M, Hurst RT, and Tajik AJ. 2010. Noninvasive measurement of central vascular pressures with arterial tonometry: clinical revival of the pulse pressure waveform? Mayo Clin Proc 85:460-472. 10.4065/mcp.2009.0336

411 Peyton PJ, and Chong SW. 2010. Minimally invasive measurement of cardiac output during surgery and critical care: a meta-analysis of accuracy and precision.

414 Porter TR, Shillcutt SK, Adams MS, Desjardins G, Glas KE, Olson JJ, and Troughton RW. 2015. Guidelines for the use of echocardiography as a monitor for 
416

417

418

419

420

421

422

423

424

425

426

427

428

430

431

432

433

434

435

436

437

438

therapeutic intervention in adults: a report from the American Society of

Echocardiography. J Am Soc Echocardiogr 28:40-56.

10.1016/j.echo.2014.09.009

Rajaram SS, Desai NK, Kalra A, Gajera M, Cavanaugh SK, Brampton W, Young D, Harvey S, and Rowan K. 2013. Pulmonary artery catheters for adult patients in intensive care. Cochrane Database Syst Rev:Cd003408.

10.1002/14651858.CD003408.pub3

Romagnoli S, Ricci Z, Romano SM, Dimizio F, Bonicolini E, Quattrone D, and De Gaudio R. 2013. FloTrac/Vigileo(TM) (third generation) and MostCare((R))/PRAM versus echocardiography for cardiac output estimation in vascular surgery. J Cardiothorac Vasc Anesth 27:1114-1121.

10.1053/j.jvca.2013.04.017

Sato T, Nishinaga M, Kawamoto A, Ozawa T, and Takatsuji H. 1993. Accuracy of a continuous blood pressure monitor based on arterial tonometry. Hypertension $21: 866-874$

Saugel B, and Reuter DA. 2014. Are we ready for the age of non-invasive haemodynamic monitoring? Br J Anaesth 113:340-343. 10.1093/bja/aeu145

Scolletta S, Franchi F, Romagnoli S, Carla R, Donati A, Fabbri LP, Forfori F, AlonsoInigo JM, Laviola S, Mangani V, Maj G, Martinelli G, Mirabella L, Morelli A, Persona P, and Payen D. 2016. Comparison Between DopplerEchocardiography and Uncalibrated Pulse Contour Method for Cardiac Output Measurement: A Multicenter Observational Study. Critical Care Medicine 44:1370-1379. 10.1097/ccm.0000000000001663 
439 Shin KY, Jeon SC, Nam KC, and Huh Y. 2010. Implementation of array sensor module

440 for a radial artery tonometry. Conf Proc IEEE Eng Med Biol Soc 2010:6397-6400. 10.1109/iembs.2010.5627310

442

444

445

446

447

448

Singh P, Choudhury MI, Roy S, and Prasad A. 2017. Computational study to investigate effect of tonometer geometry and patient-specific variability on radial artery tonometry. J Biomech 58:105-113. 10.1016/j.jbiomech.2017.04.023

Thiele RH, Bartels K, and Gan TJ. 2015. Cardiac output monitoring: a contemporary assessment and review. Critical Care Medicine 43:177-185. $10.1097 / \mathrm{ccm} .0000000000000608$

Vincent JL, Rhodes A, Perel A, Martin GS, Della Rocca G, Vallet B, Pinsky MR, Hofer CK, Teboul JL, de Boode WP, Scolletta S, Vieillard-Baron A, De Backer D, Walley KR, Maggiorini M, and Singer M. 2011. Clinical review: Update on hemodynamic monitoring--a consensus of 16. Crit Care 15:229. $10.1186 / \mathrm{cc} 10291$

Wagner JY, Langemann M, Schon G, Kluge S, Reuter DA, and Saugel B. 2016. Autocalibrating pulse contour analysis based on radial artery applanation tonometry for continuous non-invasive cardiac output monitoring in intensive care unit patients after major gastrointestinal surgery--a prospective method comparison study. Anaesth Intensive Care 44:340-345.

Wagner JY, Sarwari H, Schon G, Kubik M, Kluge S, Reichenspurner H, Reuter DA, and Saugel B. 2015. Radial Artery Applanation Tonometry for Continuous Noninvasive Cardiac Output Measurement: A Comparison With Intermittent 
461 Pulmonary Artery Thermodilution in Patients After Cardiothoracic Surgery.

$462 \quad$ Critical Care Medicine 43:1423-1428. 10.1097/ccm.0000000000000979

463 Wheeler AP, Bernard GR, Thompson BT, Schoenfeld D, Wiedemann HP, deBoisblanc

464 B, Connors AF, Jr., Hite RD, and Harabin AL. 2006. Pulmonary-artery versus

465 central venous catheter to guide treatment of acute lung injury. N Engl J Med

$466 \quad 354: 2213-2224.10 .1056 / N E J M o a 061895$

467

468

469 
472 Figure legends:

473 Figure 1. Study Flow chart. LVEF: Left ventricular ejection fraction; PAD: Peripheral 474 artery disease; TTE: transthoracic echocardiography.

475

476 Figure 2. DMP-Life System. A) Dorsal view of the DMP-life system, with the array

477 sensor in the middle of the housing. The arrows indicate the position of the 5

478 piezoresistive sensors. B) Cell diagram of the semiconductor pressure sensor: DMP-

479 Life has multi cells in one sensor tip, one sensor cell has 4 resistance materials (R1, R2, 480 R3, R4). 1-6 are connecting point of resistance material. One sensor cell gives output of 481 one pressure result. C) Schematic illustration of the sensor with the actuator while 482 applying the pressure slowly on the radial artery to detect the optimal required pressure. 483 D) Demonstrate how is the DMP-Life positioned on the left wrist. For further description 484 please refer to the main document under measurements with the DMP-life system. 485

Figure 3. Exemplary demonstration of the range of the applied pressure and the corresponding vessels response. Each yellow dot is the value of pulse height vs. applied pressure. The yellow dots make a typical shape of vessel response for each patient. Sharp end or arrow shape (like triangle) may occur in healthy elastic vessel (A,

490 B and D), but smooth and flat curved end similar to half-arc shape may result due to stiff 491 and tensed vessel (C). 
492 The applied pressure differs in each patient according to the vessels response to the

493 applied pressure. A-D demonstrate the detection of the maximum pulse height at

494 different applied pressure for each person (in A: maximum pulse height is reached when

495 applying 150 g.f/cm², in B: when applying 150 g.f/cm², in C: maximum reach when

496 applying $125 \mathrm{~g} . \mathrm{f} / \mathrm{cm}^{2}$, in D: when applying $260 \mathrm{~g} . \mathrm{f} / \mathrm{cm}^{2}$. E shows full measured pulse

497 data with stable baseline. Red rectangle shows the average range of applied pressure

498 and the corresponding pulse pressure changes in healthy people. The red arrow

499 indicates the increase of applied pressure (from right to left).

500

501

502

503 Figure 4. Exemplary demonstration of the results on the DMP-life monitor.

504 A) When the optimal signal is available, the DMP-life monitor provides a continuous 505 arterial waveform.

506 B) The DMP-life system's monitor displays inter alia CO and SV values derived from

507 pulse contour analysis. ESV: estimated stroke Volume (ESV); ESVI: estimated stroke

508 volume index; ECO: estimated cardiac output; ECRI: estimated systemic circulation

509 resistance index; SBP: systolic blood pressure; DBP: diastolic blood pressure.

510

511 Figure 5. Bland-Altman and Pearson's correlation plots. A) Bland-Altman plots of

512 stroke volume (SV) measurements B) Pearson's correlation of SV C) Bland-Altman

513 plots of cardiac output (CO) D) Pearson's correlation of $\mathrm{CO}$ measurements obtained

514 from DMP-life and from transthoracic echocardiography in 107 patients. The dotted 
515 horizontal green line shows the mean of the differences (= bias) between the two

516 methods, and the doted red horizontal lines show the upper and lower $95 \%$ limits of

517 agreement $(=$ bias $\pm 1.96 \times \mathrm{SD}) . r$ : Correlation coefficient; $r^{2}$ : Squared $r$ for the

518 goodness of fit.

519 


\section{Table $\mathbf{1}_{\text {(on next page) }}$ \\ Patients demographic.}

BMI: Body mass-index; EuroSCORE II: European system for cardiac operative risk evaluation. 
1 Table 1. Patients demographic.

\begin{tabular}{|c|c|}
\hline Variables & $\begin{array}{l}\text { Patients } \\
(n=107)\end{array}$ \\
\hline Age years & $67(58,73)$ \\
\hline Female n (\%) & $15(14.1 \%)$ \\
\hline $\mathrm{BMI} \mathrm{kg} / \mathrm{m}^{2}$ & $26.7(24.5,29.7)$ \\
\hline Peripheral artery disease $\mathrm{I}-\mathrm{II}^{\circ} \mathrm{n}(\%)$ & $22(20.6)$ \\
\hline Diabetes mellitus n (\%) & $34(31.8)$ \\
\hline Arterial hypertension $\mathbf{n}(\%)$ & $98(91.6)$ \\
\hline Chronic obstructive lung disease $n(\%)$ & $18(16.8)$ \\
\hline Chronic kidney disease n (\%) & $20(18.7)$ \\
\hline Left ventricular ejection fraction > 50\% $n(\%)$ & $48(44.8)$ \\
\hline Left ventricular ejection fraction $40-50 \% \mathrm{n}(\%)$ & $59(55.2)$ \\
\hline Mean Heartrate per minute & $72(64,86)$ \\
\hline Mean systolic pressure mmHg & $134(128,147)$ \\
\hline Mean diastolic pressure $\mathrm{mmHg}$ & $83(78,87)$ \\
\hline EuroSCORE II \% & $3.4 \pm 1.9$ \\
\hline
\end{tabular}

2 BMI: Body mass-index; EuroSCORE II: European System

3 for Cardiac Operative Risk Evaluation 


\section{Figure 1 (on next page)}

Study flow chart.

LVEF: Left ventricular ejection fraction; PAD: Peripheral artery disease; TTE: transthoracic echocardiography. 
Between January 2015 and December 2016

572 patients were screened

Patients excluded due:

$>$ Moderate to severe aortic valve stenosis $(n=88)$

$>$ Moderate to severe aortic regurgitation $(n=26)$

$>$ Moderate to severe Mitral regurgitation $(n=46)$

$>\operatorname{LVEF}<40 \%(n=104)$

$>\mathrm{PAD}>\mathrm{II}^{\circ}(\mathrm{n}=84)$

$>$ Atrial fibrillation $(n=57)$

$>$ Inaccurate acoustic Window for TTE $(n=27)$

$>$ Participate in other Study $(n=33)$

107 patients enrolled in the pilot study 


\section{Figure 2}

DMP-Life System.

A) Dorsal view of the DMP-life system, with the array sensor in the middle of the housing. The arrows indicate the position of the 5 piezoresistive sensors. B) Cell diagram of the semiconductor pressure sensor: DMP-Life has multi cells in one sensor tip, one sensor cell has 4 resistance materials ( $R 1, R 2, R 3, R 4)$. 1-6 are connecting point of resistance material. One sensor cell gives output of one pressure result. C) Schematic illustration of the sensor with the actuator while applying the pressure slowly on the radial artery to detect the optimal required pressure. D) Demonstrate how is the DMP-Life positioned on the left wrist. For further description please refer to the main document under measurements with the DMP-life system.

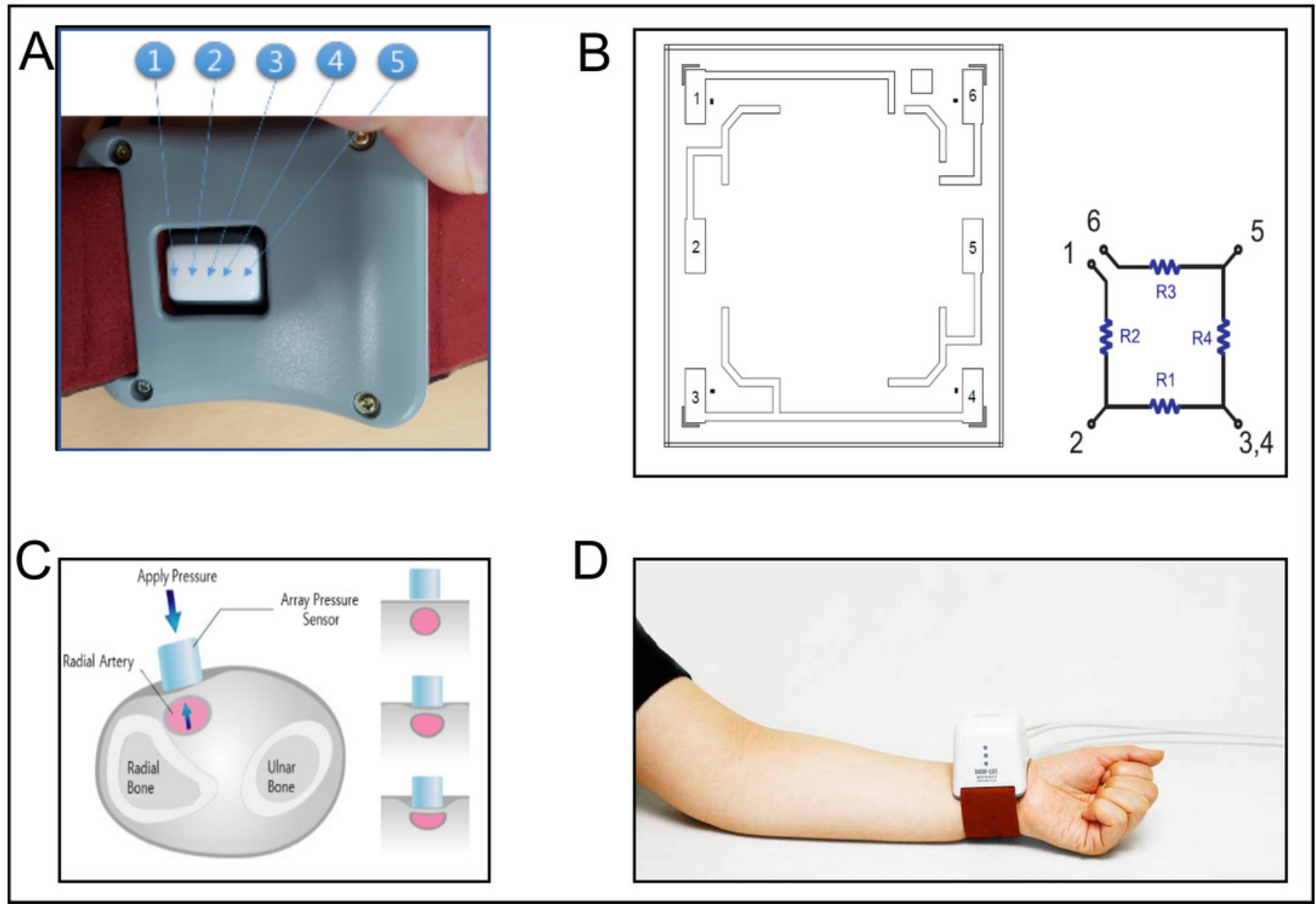




\section{Figure 3}

Exemplary demonstration of the range of the applied pressure and the corresponding vessels response.

Each yellow dot is the value of pulse height vs. applied pressure. The yellow dots make a typical shape of vessel response for each patient. Sharp end or arrow shape (like triangle) may occur in healthy elastic vessel (A, B and D), but smooth and flat curved end similar to half-arc shape may result due to stiff and tensed vessel (C). The applied pressure differs in each patient according to the vessels response to the applied pressure. A-D demonstrate the detection of the maximum pulse height at different applied pressure for each person (in A: maximum pulse height is reached when applying $150 \mathrm{~g} . \mathrm{f} / \mathrm{cm}^{2}$, in $\mathrm{B}$ : when applying 150 g.f $/ \mathrm{cm}^{2}$, in C: maximum reach when applying $125 \mathrm{~g} . \mathrm{f} / \mathrm{cm}^{2}$, in D: when applying $260 \mathrm{~g} . \mathrm{f} / \mathrm{cm}^{2}$. E shows full measured pulse data with stable baseline. Red rectangle shows the average range of applied pressure and the corresponding pulse pressure changes in healthy people. The red arrow indicates the increase of applied pressure (from right to left).
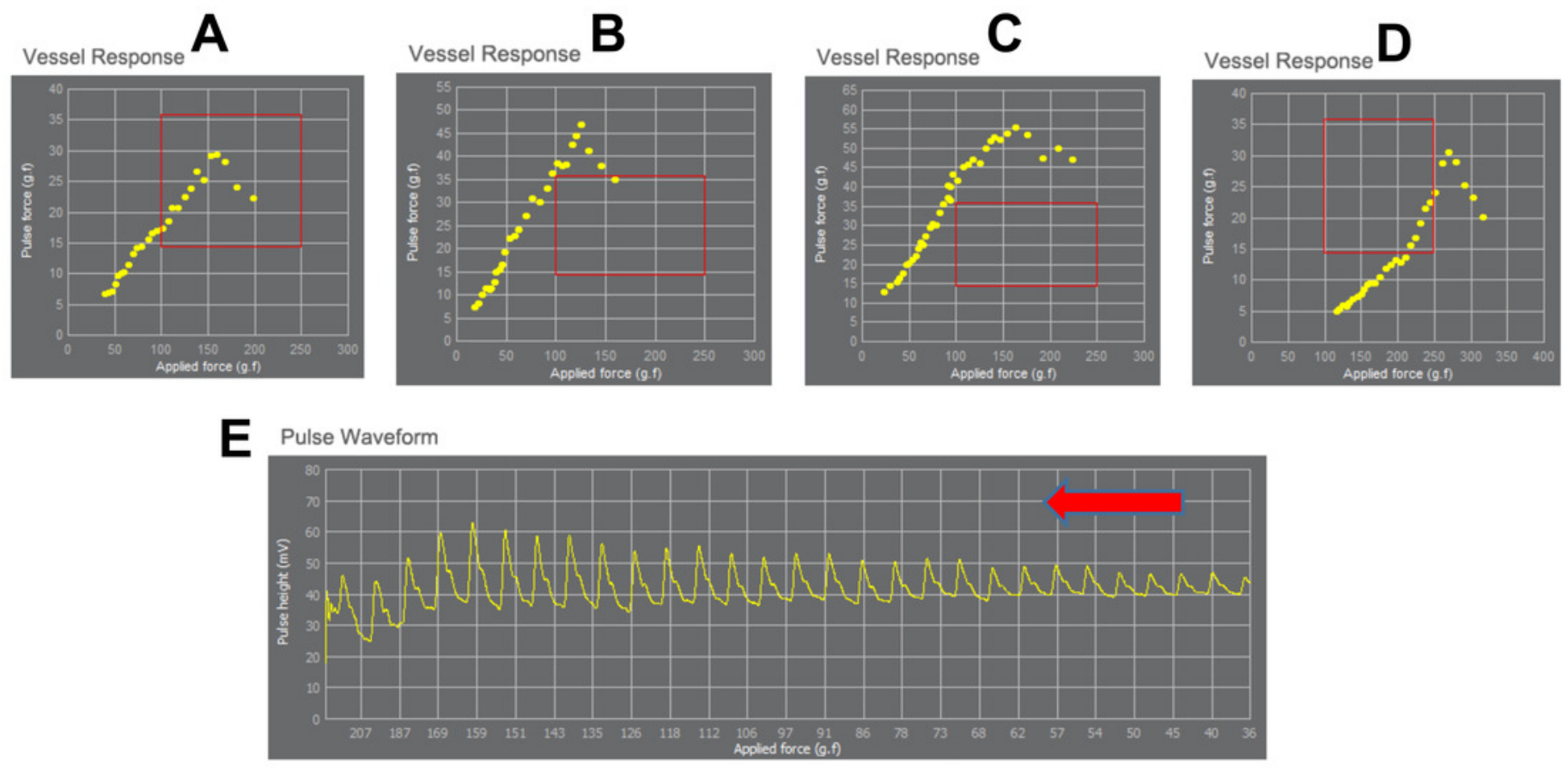


\section{Figure 4}

Exemplary demonstration of the results on the DMP-life monitor.

A) When the optimal signal is available, the DMP-life monitor provides a continuous arterial waveform. B) The DMP-life system's monitor displays inter alia CO and SV values derived from pulse contour analysis. ESV: estimated stroke Volume (ESV); ESVI: estimated stroke volume index; ECO: estimated cardiac output; ECRI: estimated systemic circulation resistance index; SBP: systolic blood pressure; DBP: diastolic blood pressure. 

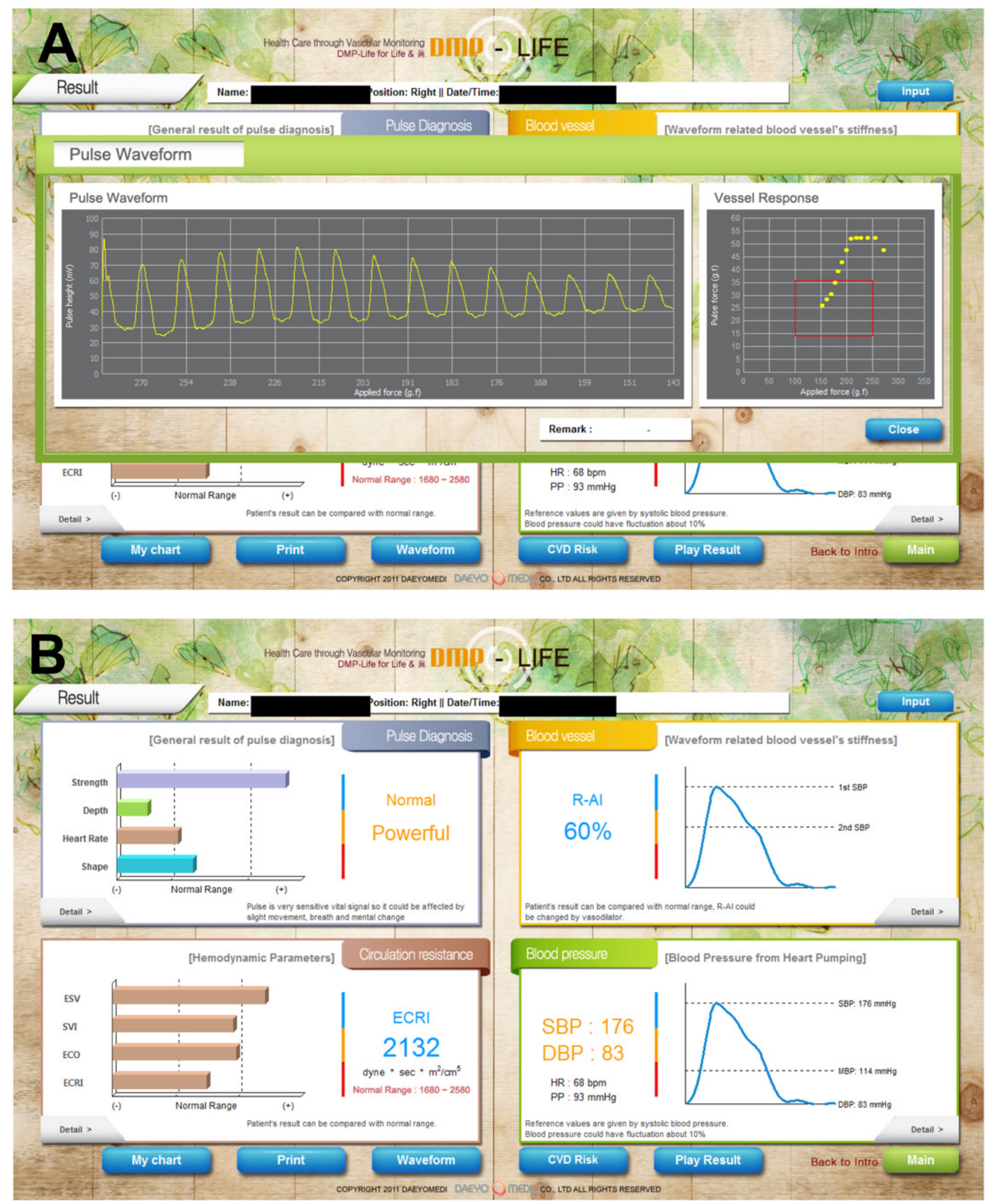
Figure $\mathbf{5}$ (on next page)

Bland-Altman and Pearson's correlation plots.

A) Bland-Altman plots of stroke volume (SV) measurements B) Pearson's correlation of SV C) Bland-Altman plots of cardiac output (CO) D) Pearson's correlation of CO measurements obtained from DMP-life and from transthoracic echocardiography in 107 patients. The dotted horizontal green line shows the mean of the differences (= bias) between the two methods, and the doted red horizontal lines show the upper and lower $95 \%$ limits of agreement ( $=$ bias $\pm 1.96 \times$ SD). $r$ : Correlation coefficient; $r^{2}$ : Squared $r$ for the goodness of fit. 
A Bland-Altman of sv

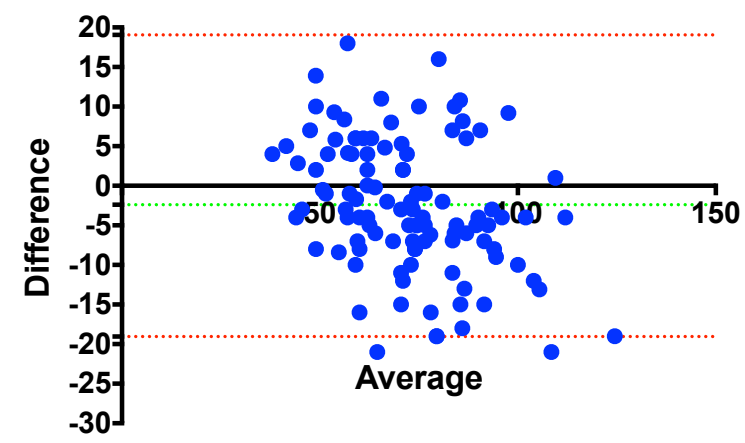

C

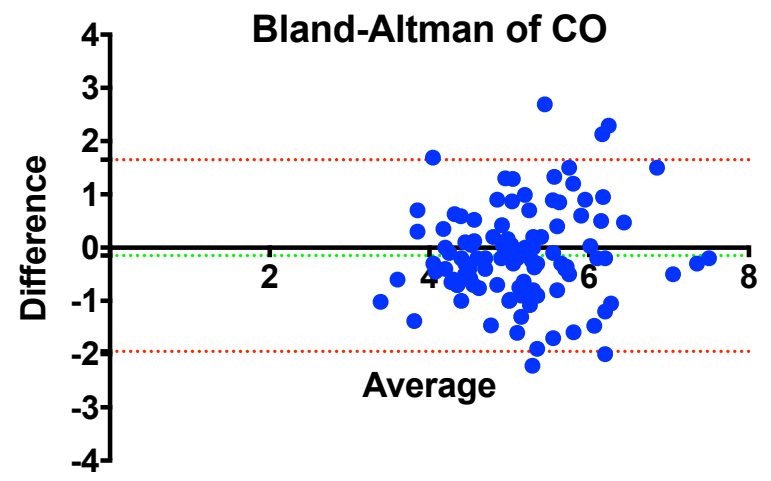

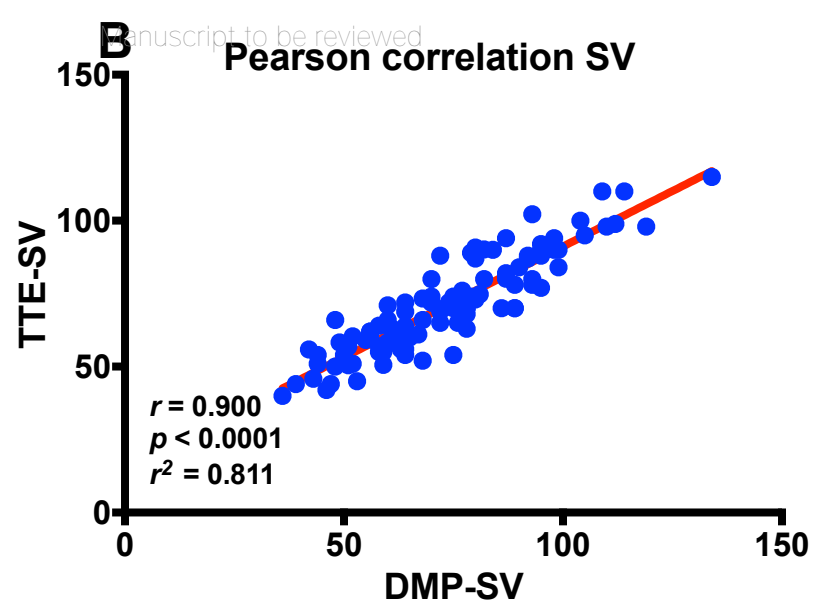

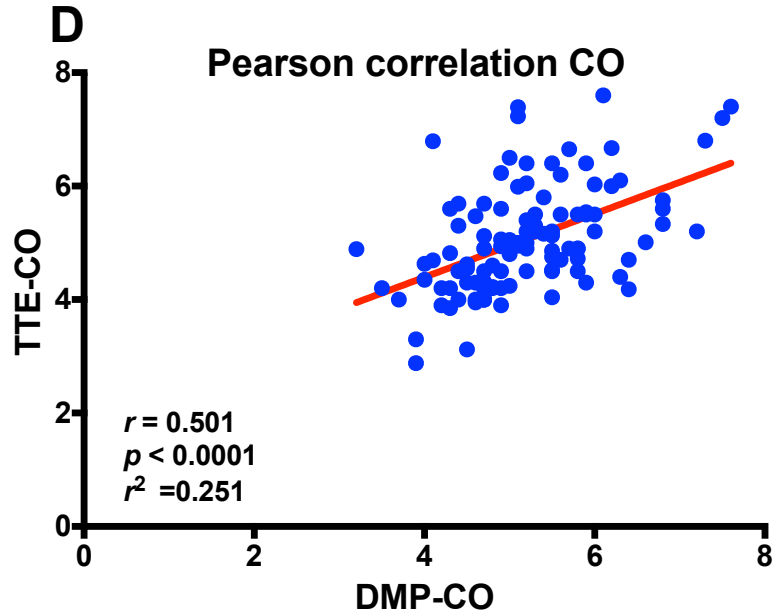

